\title{
Development of Removable Hollow Cathode Discharge Apparatus for Sputtering Solid Metals
}

\author{
Daisuke Ishikawa $\mathbb{I D}^{1}$ and Shuichi Hasegawa $\mathbb{i D}^{1,2}$ \\ ${ }^{1}$ Department of Nuclear Engineering and Management, University of Tokyo, 7-3-1 Hongo, Bunkyo-ku, \\ Tokyo 113-0033, Japan \\ ${ }^{2}$ Nuclear Professional School, University of Tokyo, 2-22 Shirane, Shirakata, Tokai, Naka, Ibaraki 319-1188, Japan \\ Correspondence should be addressed to Daisuke Ishikawa; ishikawa@lyman.q.t.u-tokyo.ac.jp
}

Received 12 February 2019; Revised 25 March 2019; Accepted 1 April 2019; Published 17 April 2019

Academic Editor: Nikša Krstulović

Copyright @ 2019 Daisuke Ishikawa and Shuichi Hasegawa. This is an open access article distributed under the Creative Commons Attribution License, which permits unrestricted use, distribution, and reproduction in any medium, provided the original work is properly cited.

\begin{abstract}
A hollow cathode discharge cell has been developed for a variety of sample phases. To make the experimental apparatus compact, we have adopted DC discharge, and the discharge cell was inexpensively constructed for replacement with commercially available vacuum parts. The most distinctive attribute of the developed cell is the double structure of the cathodes. The occurrence of sputtering resulted in the metal atom in gas phase. With Ar buffer gas, the favorable condition of laser absorption spectroscopy for the gas phase sample was investigated.
\end{abstract}

\section{Introduction}

A hollow cathode concentrates electron density in the center of the cathode. As a result, it is expected that in this region, the density of excited atomic species is highest [1]. This geometry is suitable for laser spectroscopy, and consequently, hollow cathode discharge cells have already been made commercially available [2]. Paschen first developed this structure and Jones and Walsh [3] and Grimm [4] improved it for atomic absorption spectroscopy, in particular for the purpose of isotope spectroscopy. In addition, many articles referencing the technique have been published, such as in laser frequency calibration and absorption spectroscopy of Li [5], Fe [6], U [7], and molecules [8]. The excited noble gas atoms in discharge collide with other atoms, the transfer of energy causing excitation of electrons. Additionally, electrons in a discharge may also cause atomic excitation. It does not require multiple laser sources for excitation like in Resonance Ionization Spectroscopy (RIS). In addition, excited atomic species or ions of noble gases can be generated easily and may be used to produce a vapor of solid metal samples by way of sputtering, which is the collision of the noble gas ions with the metal electrodes resulting in the ejection of the metal atoms. Sputtering as a technique is widely used for fabrication of multithin films in material science, with particular merit being that it can produce the required atomic vapor with a minimum chemical pretreatment of the donor material surface. Therefore, the use of this method in conjunction with discharge spectroscopy provides an advantage over other popular spectroscopic methods, such as Inductively Coupled Plasma Atomic Emission Spectroscopy (ICP-AES), which do require extensive pretreatment. Various methods are utilized for vaporizing samples such as direct current (DC) [9], radio frequency (rf) [10], and microwave discharges [11]. Mass spectrometry like Secondary Ion Mass Spectrometry (SIMS) [12] makes use of sputtering for vaporization of a sample. The experimental setup required is generally large; however, DC sputtering is small and low cost.

In this work, we describe the development of a hollow cathode glow discharge cell capable of analyzing a variety of samples, a property which is difficult for commercially available items to achieve because of their sealed structure. There are a few publications [5-8] reporting discharge cells capable of the analysis of one specific element. However, our discharge cell may be able to be applied to the analysis of 
multiple samples/elements thanks to the double cathode structure, wherein the inner cathode is demountable. Our discharge cell is expected to be able to analyze not only gases, but also liquid residues on the electrode, and solid metals used as the electrode in bulk form. In addition, the memory effect (effects of the residues of previous experiments) can be disregarded in applying the apparatus to chemical analysis, due to the replaceability of the inner cathode. As examples of the apparatus capability in regard to solid metals, a tungsten hollow cathode and liquid residue, mixed elements ICP standard sample, are shown to be effective as a metal vapor source without additional chemical pretreatment. Subsequently, the various isotopes of the excited noble gas could be observed in spectroscopy with a high-resolution CW laser. Out of the various kinds of discharge techniques, we employed DC glow discharge. Glow discharge takes place in low-pressure plasmas where the temperature of the ions is different from that of the electrons. It is less damaging to components than arc discharge, and the noble gas density for inducing discharge is low, which is ideal for optical spectroscopy.

\section{Experimental Setup}

The discharge cell was designed to consist of an electrically insulated, transparent Duran NW40 cross (Evac, 33.040007.171.740). For spectroscopy, low-absorption transparent view ports (Evac, 32.123.840) were employed. An electric current feed through flange (KyoCera, N405BR2) for two MHV terminals with long ceramic insulators to avoid inappropriate discharge between the feed through and the flange was used to introduce the electrical connections. The pressure in the cell was monitored with a capacitance gauge (Canon-Anelva, M-342DG-13) with range from $10 \mathrm{~Pa}$ to atmospheric pressure. Air was evacuated by a rotary pump (Pfeiffer Vacuum, 2005 SD), and the discharge buffer gas (Ar or Ne) was supplied via a bellows valve (Swagelok, SS-4BG). Isolation of the cell from the pumping pipeline was achieved by a diaphragm valve (VAT, 22032-KA01) (Figure 1). The cathode electrodes have a doubled structure (Figure 2). This structure enables the investigation of various samples simply by changing the inner cathode. They are made of titanium (inner) and nichrome (outer), and their sizes are as follows: inner cathode (right upwarddashed in Figure 2, TI-45590, purchased from Nilaco, Tokyo, Japan) has $4.1 \mathrm{~mm}$ inner diameter (ID), $5.1 \mathrm{~mm}$ outer diameter (OD), and $27 \mathrm{~mm}$ in length. The dimension of the outer cathode (orange solid color in Figure 2) is $5.2 \mathrm{~mm}$ (ID), $10 \mathrm{~mm}$ (OD), and $27 \mathrm{~mm}$ in length. The cathode edges were not rounded to prevent surface discharge. Titanium was employed because of its low cost and high heat durability. Nichrome was employed because of its higher durability against discharge ablation compared to stainless steel, based on our experience. The external surface of the hollow cathode (left upward-dashed in Figure 2) is insulated by glass. The dimensions of the glass components are ID $(10.2 \mathrm{~mm})$, OD $(14 \mathrm{~mm})$, and the length $(28 \mathrm{~mm})$. The glass is supported by the cathode

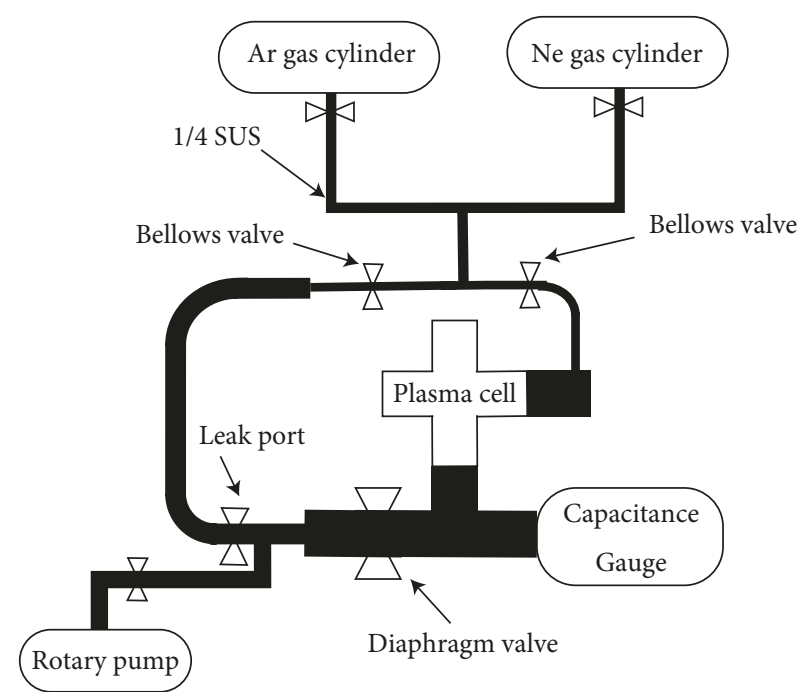

Figure 1: Diagram of the plasma experimental setup.

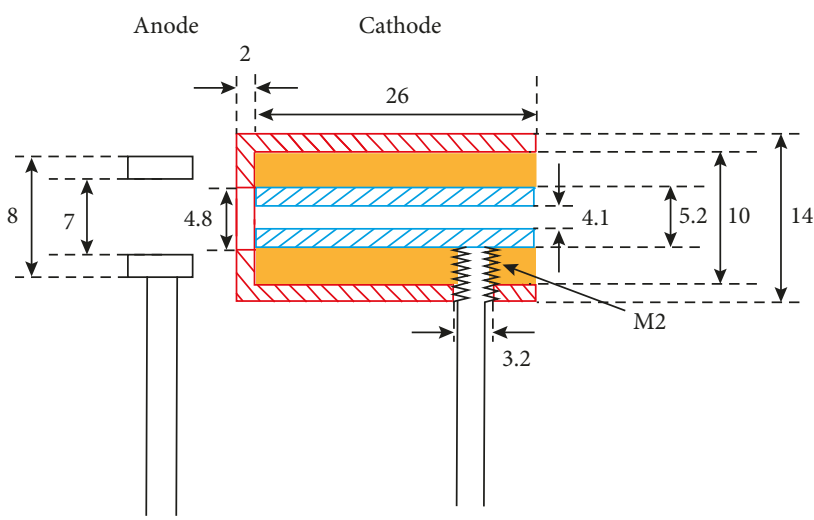

Figure 2: Schematic of the electrode system (sizes in $\mathrm{mm}$ ).

standing rod. The anode is made of stainless steel and its size is $7 \mathrm{~mm}$ (ID), $8 \mathrm{~mm}$ (OD). The distance between the electrodes is approximately $3-4 \mathrm{~mm}$. To allow for continuous discharge, a ballast resistor of $1 \mathrm{k} \Omega$ is connected to the plasma cell in series. A voltmeter was also connected across the resistor to monitor the resistor voltage. The voltage between the electrodes was calculated from the voltage of the electronic power supply minus that of the ballast resistor. The specification of the power supply is $360 \mathrm{~W}, 800 \mathrm{~V}$ (Texio PSW360-800H).

A Ti:S laser (Coherent, 899-21) excited by a diode pumped solid-state laser (Coherent, Verdi10) was employed for laser absorption spectroscopy (Figure 3). Frequency calibration was achieved with a Fizeau type interference wavemeter (High Finesse, WSU-30). Light transmitted through the cell was observed with a photodetector. High-sensitivity detection was obtained by a Lock-in-Amplifier (Stanford Research Systems, SR830) and optical-chopper (Stanford Research Systems, SR540). For emission spectroscopy, a compact spectrometer (Ocean Optics, Flame-S, wavelength resolution: $1.02 \mathrm{~nm}$, observable range: $190-880 \mathrm{~nm}$, cumulative time: $1 \mathrm{~ms}-$ 


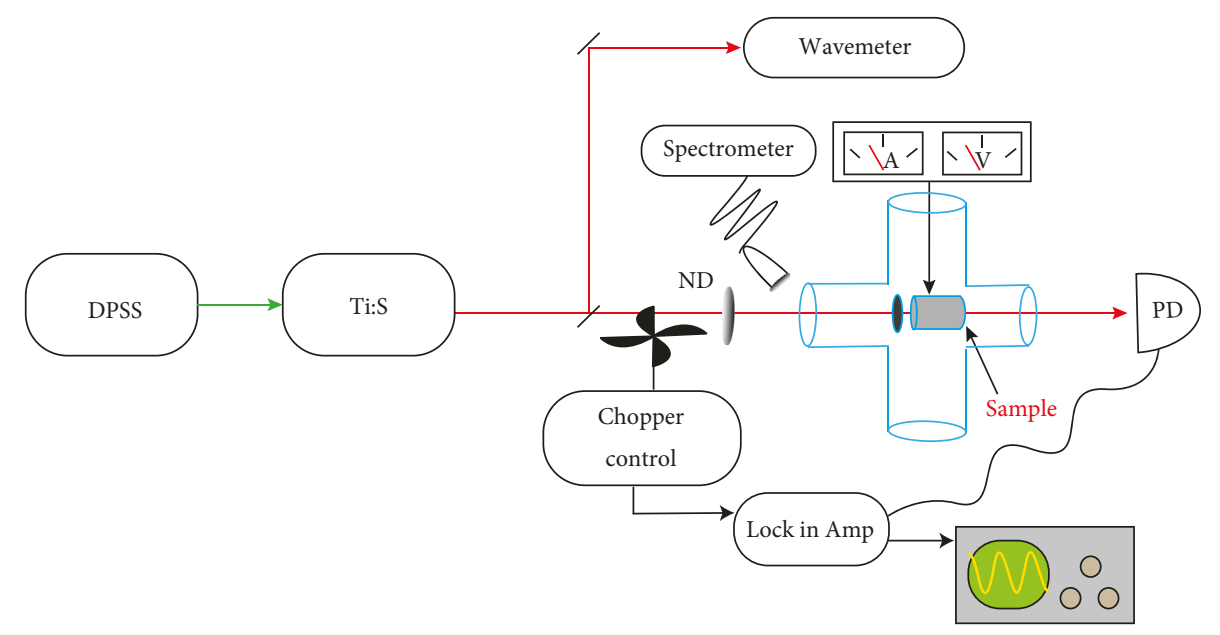

Figure 3: Schematic diagram of the experimental setup.

$65 \mathrm{~s})$ was used to monitor chemical species, such as atoms and molecules, in the discharge. The control and data acquisition was conducted via LabVIEW (National Instruments) and VBA (Microsoft).

\section{Results}

Sputtering is efficient in the abnormal glow region [13]. With this method, solid samples may be vaporized into single atoms even if they are present in the sample as chemical compound constituents. As examples, the emission spectra of sputtering of solid tungsten (Figure 4) and liquid residue containing several elements (Figure 5) were observed. The liquid residue sample was dropped onto the electrode and dried with infrared lamp heating. Thanks to this technique, metals of nonneutral atom were made into those of neutral atom without chemical reduction treatment. Although the elemental assignment of the mixed sample (Merck, ICP multielement standard IV) was successful on 17 elements, through comparison with the observed spectrum of pure element or spectra database [14], 6 elements $\mathrm{Ag}, \mathrm{Bi}, \mathrm{Cd}, \mathrm{Co}, \mathrm{Li}$, and $\mathrm{Tl}$ were not able to be assigned, due to the interference with other signals. As an example, in the case of $\mathrm{Li}$, the wavelength of the distinctive peaks is $670.776 \mathrm{~nm}$ and $670.791 \mathrm{~nm}$, but there are emissions close to these values: $\mathrm{Ne}$ emission at $671.704 \mathrm{~nm}$ and Ar at $669.887 \mathrm{~nm}$. Therefore, a spectrometer resolution of less than $0.5 \mathrm{~nm}$ would be required to resolve the Li signals. As Figure 5 shows, the metals in liquid residue may exist as the neutral atoms, even if their metal valence are between one and three in solution.

The transitions used in the laser absorption spectroscopy experiment are $2 \mathrm{p}^{5}\left({ }^{2} \mathrm{P}_{3 / 2}^{\mathrm{O}}\right) 3 \mathrm{~s}^{2}[3 / 2]^{\mathrm{O}}(J=1) \longrightarrow 2 \mathrm{p}^{5}\left({ }^{2} \mathrm{P}_{3 / 2}^{\mathrm{O}}\right)$ $3 \mathrm{p}^{2}[1 / 2] J=1$ for $\mathrm{Ne}$ and $3 \mathrm{p}^{5}\left({ }^{2} \mathrm{P}_{3 / 2}^{\mathrm{O}}\right) 4 \mathrm{~s}^{2}[3 / 2]^{\mathrm{O}} J=1 \longrightarrow 3 \mathrm{p}^{5}$ $\left({ }^{2} \mathrm{P}^{\mathrm{O}}{ }_{1 / 2}\right) 4 \mathrm{p}^{2}[3 / 2]_{2} J=2$ for Ar. Figure 6 shows the $\mathrm{Ne}$ absorption spectrum. From Voigt fitting, the isotope shift of Ne-20 (90\% abundance) and Ne-22 (10\%) was estimated to be $0.92 \mathrm{GHz}$.

The intensities of absorption for excited species of Ar were investigated in a continuous flow of Ar and also for a

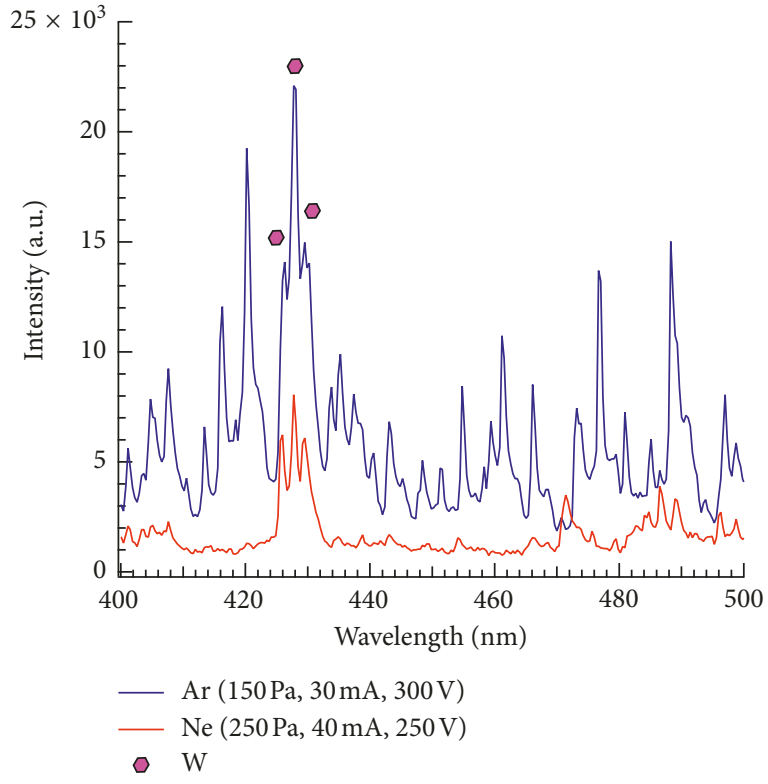

Figure 4: Tungsten hollow cathode emission spectrum.

sealed cell of Ar. The "sealed mode" was the condition under which the bellows valve and the diaphragm valve were closed. "Flow mode" is the name given to the condition where the experiment was performed with the intake produced by rotary pump and with the gas cylinder regulator open. Ar absorbance was measured in pressure range $100-400 \mathrm{~Pa}$ with $20 \mathrm{~mA}$ (Figure 7). At less than $100 \mathrm{~Pa}$, discharge was not observed, due to the atomic density being too low for discharge to occur. In the continuous gas flow mode, atoms in the excited state are continually replaced with atoms in the ground state, due to the flow of the gas. The concentration of atoms in highly excited states at the cathode was lower in the flow mode. The ratio of atoms in the lower state to higher state is different between the flow mode and sealed mode. It resulted in larger absorbance in flow mode as shown in Figure 7. In addition, the dependency of absorbance was investigated for various pressures and currents (Figure 8). 


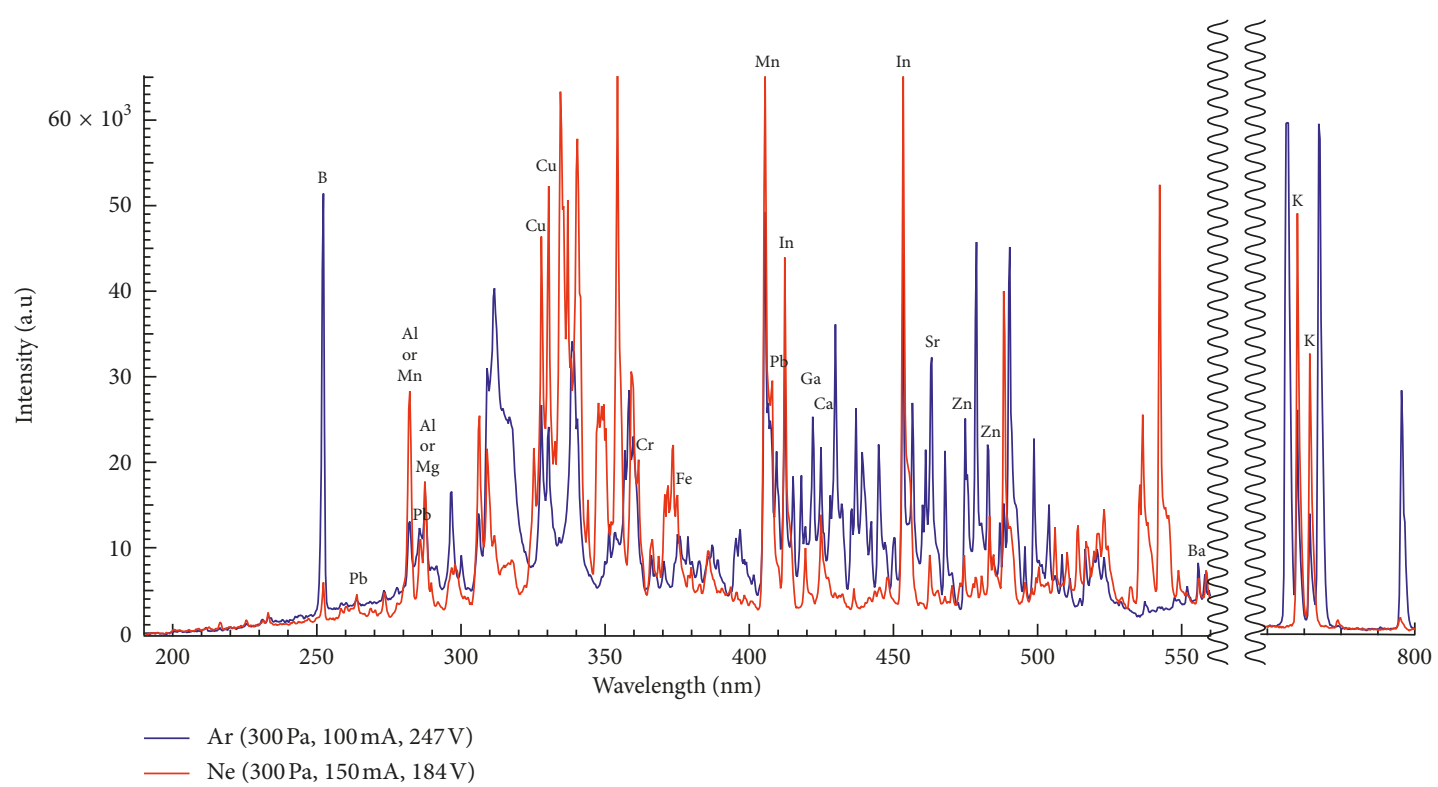

FIGURE 5: Mixed elements standard sample liquid residue emission spectrum.

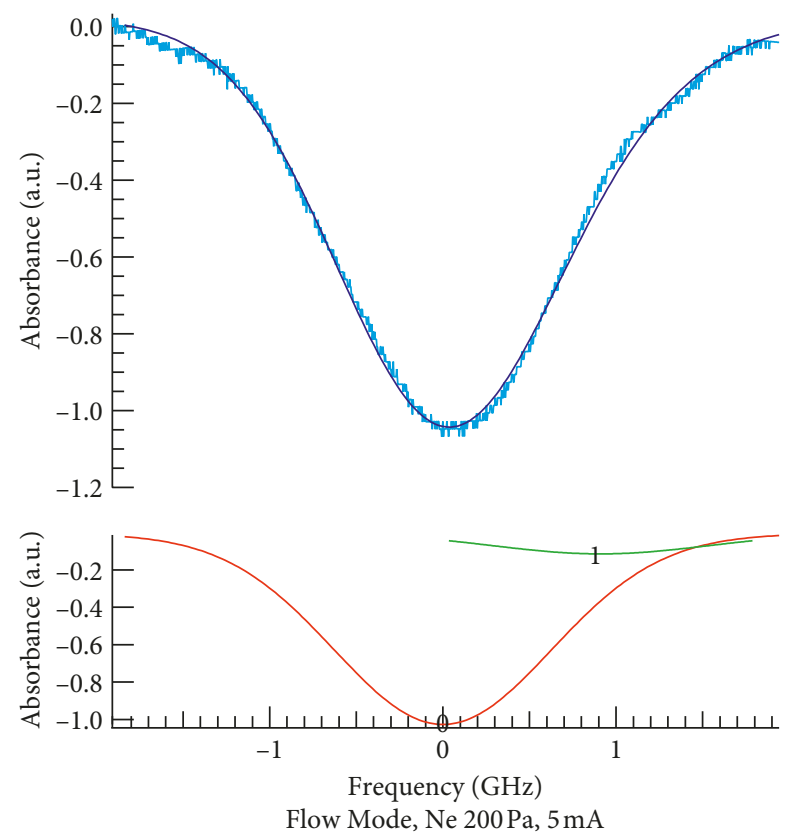

Figure 6: Ne absorption spectrum for the $2 \mathrm{p}^{5}\left({ }^{2} \mathrm{P}_{3 / 2}^{\mathrm{O}}\right) 3 \mathrm{~s}^{2}[3 / 2]^{\mathrm{O}}(J=1) \longrightarrow 2 \mathrm{p}^{5}\left({ }^{2} \mathrm{P}_{3 / 2}^{\mathrm{O}}\right) 3 \mathrm{p}^{2}[1 / 2] J=1$.

It shows higher current results in a larger absorbance. The increase of current caused an increase in electron density and therefore increased probability of the excitation of gases in discharge. From this increase in probability, the relationship between current and absorbance was subsequently identified. In addition, the increase of the pressure decreased the density of excited atomic gases. Consequently, we can conclude that the relation between gas pressure and absorbance is that absorbance decreases with pressure increase. From Figures 7 and 8, the preferable conditions for higher absorption are at lower pressure and higher current for this transition.

\section{Conclusion}

We developed a hollow cathode cell, which can be used to observe absorption and emission of noble gases with isotope shift resolution under illumination by laser light. By discharge technique, tungsten and mixed liquid residue were sputtered to atoms, which can be utilized as the neutral metal atom vapor sources. The inner cathode of the double cathode structure is demountable and can allow for the introduction of a variety of sample phases, such as gases, liquid residues, or metal solids. For a metal vapor source, preferable sputtering conditions were investigated with various pressures of $\mathrm{Ar}$ and $\mathrm{Ne}$ under 


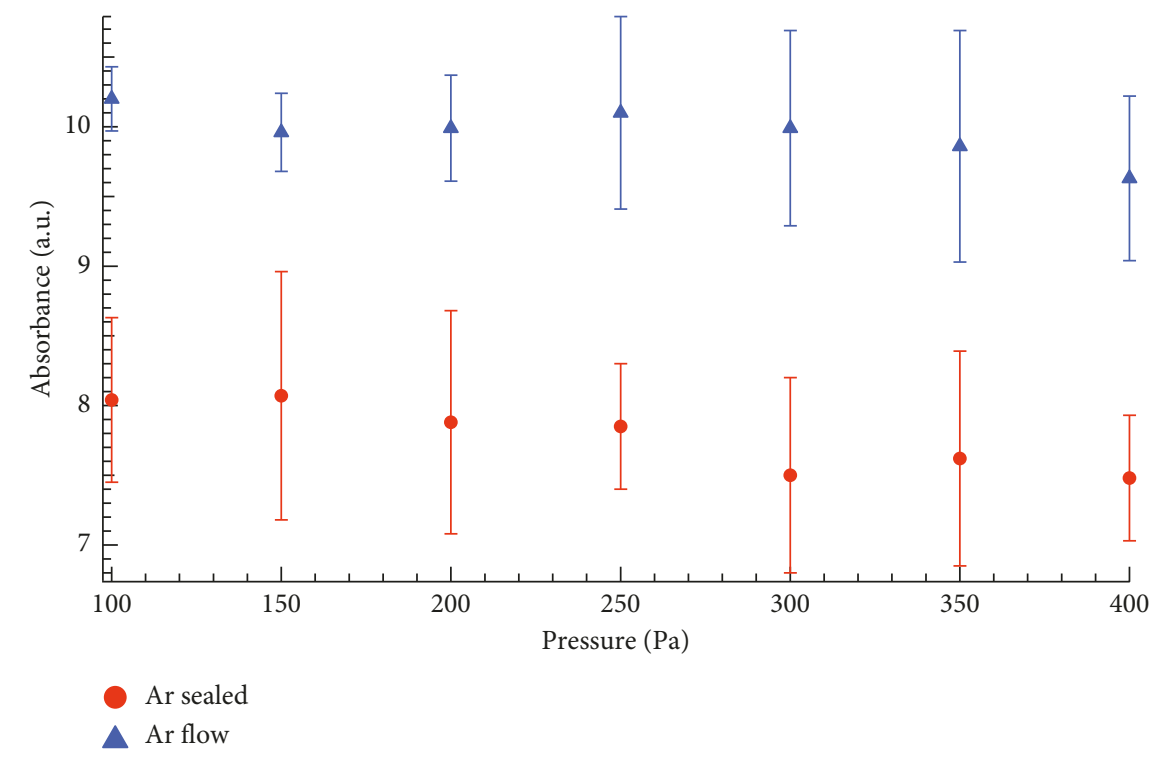

Figure 7: Absorbance of Ar in flowing gas and sealed cell cases at $20 \mathrm{~mA}$.

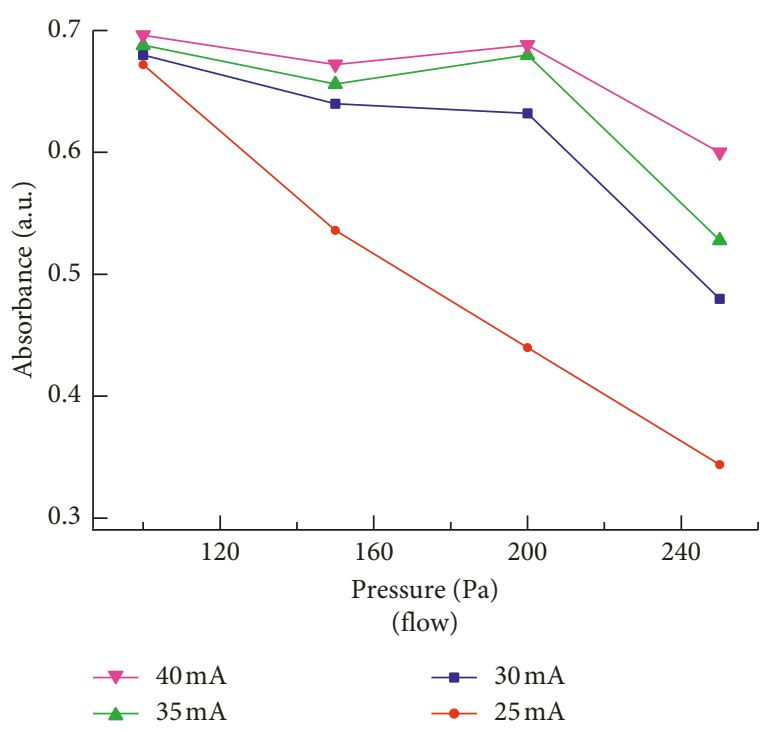

FIgURE 8: Change of absorbance of Ar under various pressures and currents in flow mode.

discharge. In the future, we intend to further investigation of laser absorption spectroscopy using the experiment of apparatus.

\section{Data Availability}

The data used to support the findings of this study are available from the corresponding author upon request.

\section{Conflicts of Interest}

The authors declare that there are no conflicts of interest related to this article.

\section{Acknowledgments}

Part of this research was supported by "HRD for Fukushima Daiichi Decommissioning based on Robotics and Nuclide Analysis" carried out under the Center of World Intelligence Project for Nuclear S\&T and Human Resource Development by the Ministry of Education, Culture, Sports, Sciences and Technology (MEXT), Japan. The authors would like to thank S. R. Wells for proofreading of the manuscript.

\section{References}

[1] S. Muhl and A. Pérez, "The use of hollow cathodes in deposition processes: a critical review," Thin Solid Films, vol. 579, pp. 174-198, 2015.

[2] Y. Hayakawa, T. Sato, C. Watanabe, T. Aoki, and Y. Torii, "Doppler-free spectroscopy of metastable Sr atoms using a hollow cathode lamp," Applied Optics, vol. 57, no. 6, pp. 1450-1454, 2018.

[3] W. G. Jones and A. Walsh, "Hollow-cathode discharges-the construction and characteristics of sealed-off tubes for use as spectroscopic light sources," Spectrochimica Acta, vol. 16, no. 3, pp. 249-254, 1960.

[4] W. Grimm, "Eine neue glimmentladungslampe für die optische emissionsspektralanalyse," Spectrochimica Acta Part B: Atomic Spectroscopy, vol. 23, no. 7, pp. 443-454, 1968.

[5] V. K. Saini, P. Kumar, K. K. Sarangpani, S. K. Dixit, and S. V. Nakhe, "Development of a see-through hollow cathode discharge lamp for ( $\mathrm{Li} / \mathrm{Ne})$ optogalvanic studies," Review of Scientific Instruments, vol. 88, no. 9, article 093101, 2017.

[6] B. Smeets, R. C. M. Bosch, P. van der Straten et al., "Laser frequency stabilization using an Fe-Ar hollow cathode discharge cell," Applied Physics B, vol. 76, no. 8, pp. 815-819, 2003.

[7] C. M. Barshick, R. W. Shaw, J. P. Young, and J. M. Ramsey, "Evaluation of the precision and accuracy of a uranium isotopic analysis using glow discharge optogalvanic spectroscopy," Analytical Chemistry, vol. 67, no. 20, pp. 38143818, 1995. 
[8] F. C. Van Den Heuvel and A. Dymanus, "Observation of farinfrared transitions of $\mathrm{HCO}^{+}, \mathrm{CO}^{+}$and $\mathrm{HN}_{2}^{+}$," Chemical Physics Letters, vol. 92, no. 3, pp. 219-222, 1982.

[9] P. Jacquet and A. Pailloux, "Laser absorption spectroscopy for xenon monitoring in the cover gas of sodium cooled fast reactors," Journal of Analytical Atomic Spectrometry, vol. 28, no. 8, pp. 1298-1302, 2013.

[10] U. Kortshagen, I. Pukropski, and M. Zethoff, "Spatial variation of the electron distribution function in a rf inductively coupled plasma: experimental and theoretical study," Journal of Applied Physics, vol. 76, no. 4, pp. 2048-2058, 1994.

[11] Y. Yoshida, "Microwave-enhanced magnetron sputtering," Review of Scientific Instruments, vol. 63, no. 1, pp. 179-183, 1992.

[12] H. A. Storms, K. F. Brown, and J. D. Stein, "Evaluation of a cesium positive ion source for secondary ion mass spectrometry," Analytical Chemistry, vol. 49, no. 13, pp. 20232030, 1977.

[13] M. J. Druyvestevn and F. M. Penning, "The mechanism of electrical discharges in gases of low pressure," Reviews of Modern Physics, vol. 12, no. 2, pp. 87-174, 1940.

[14] NIST Atomic Database Spectra, https://www.nist.gov/pml/ atomic-spectra-database. 

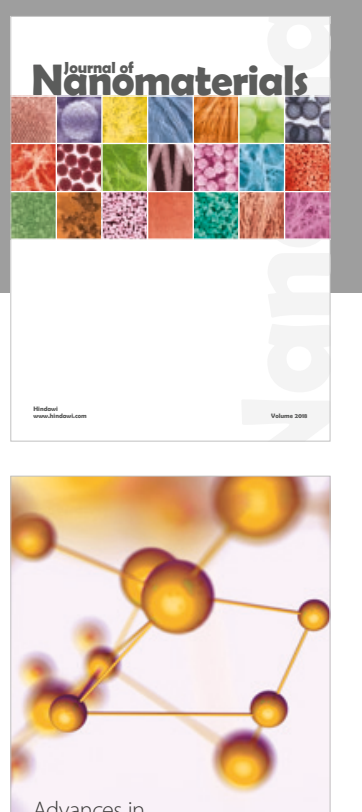

Physical Chemistry
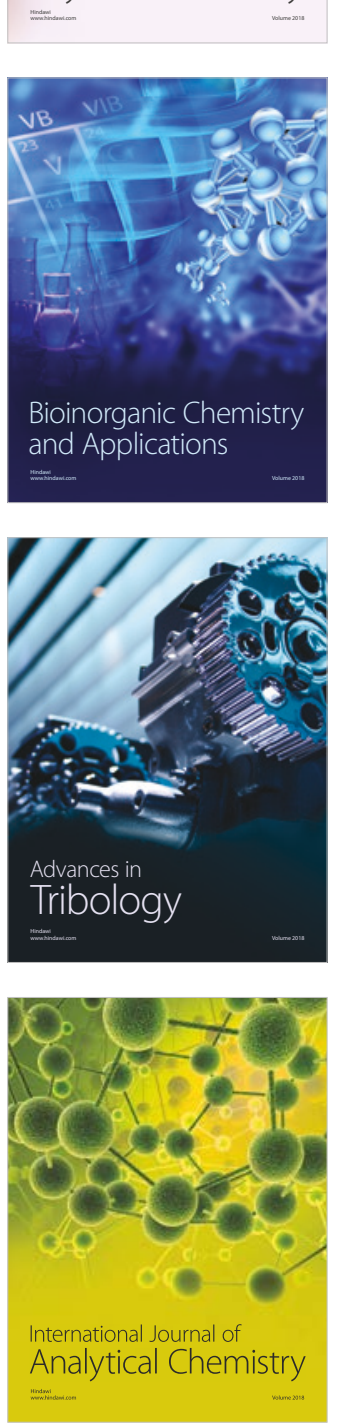

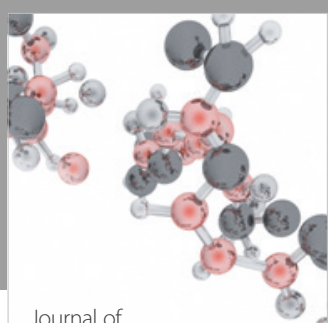

Analytical Methods

in Chemistry

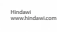

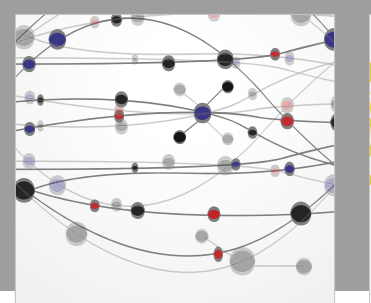

The Scientific World Journal

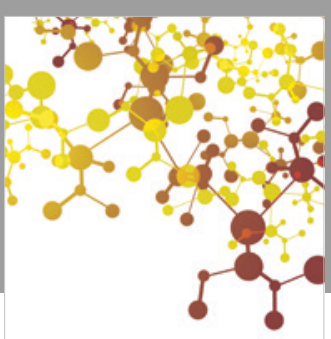

Journal of

Applied Chemistry
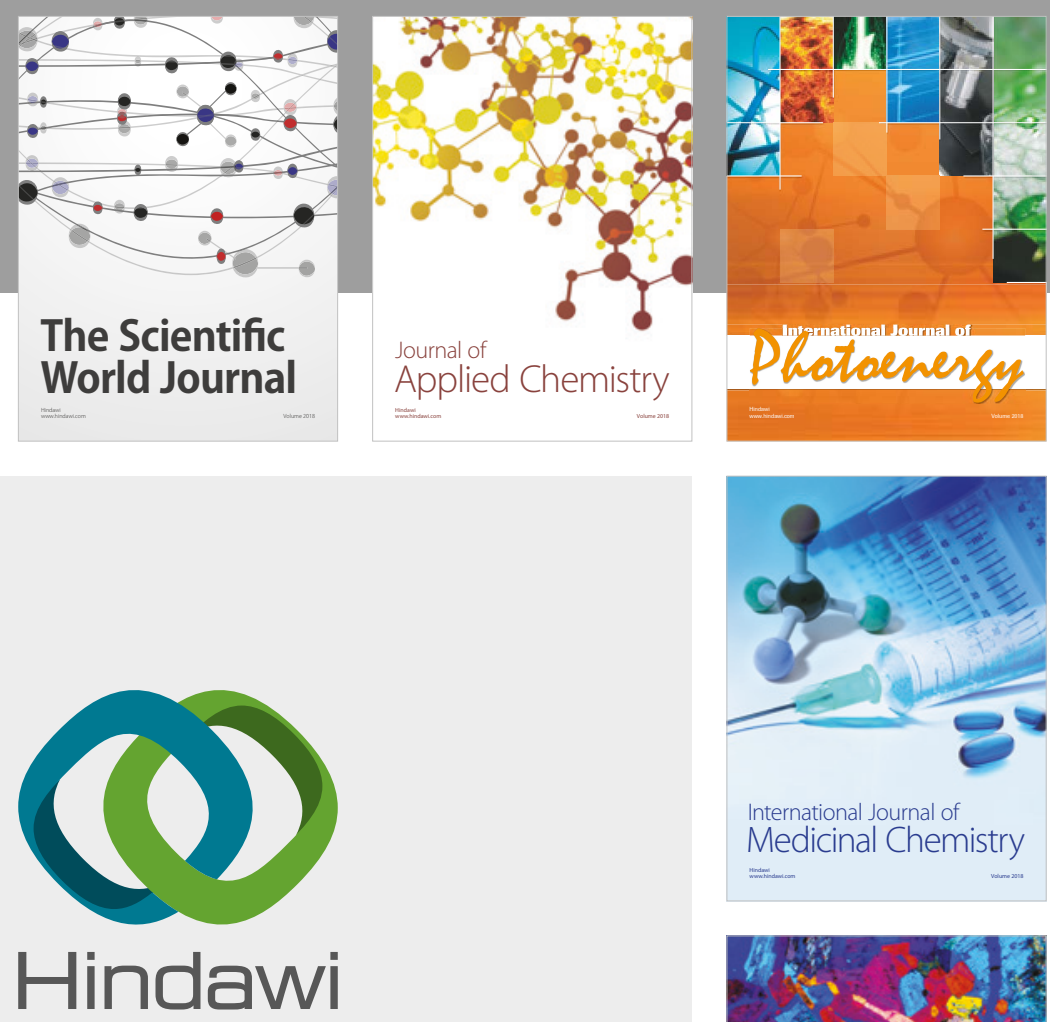

Submit your manuscripts at

www.hindawi.com
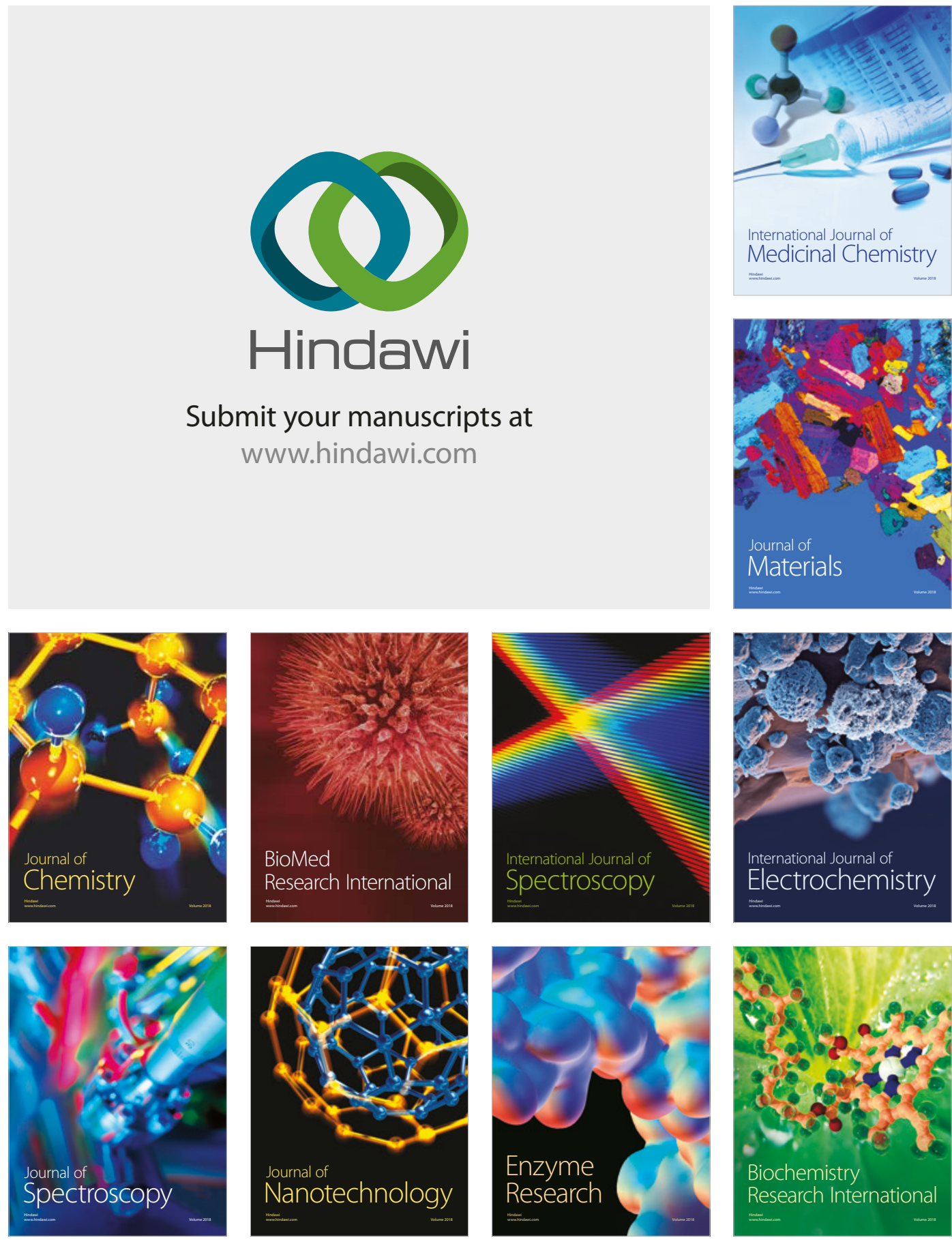
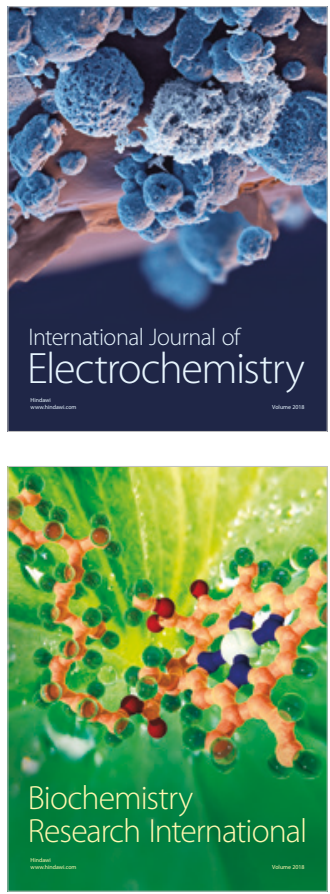